\title{
CONTRIBUTION TO THE BRYOPHYTE FLORA OF BJELASICA MTS (MONTENEGRO) ${ }^{1}$
}

\author{
BeÁta Papp, Peter ERzberger \& Snežana Dragićević
}

\begin{abstract}
During field trips made in 2007 and 2008 to the Bjelasica Mts, 318 bryophyte taxa (84 liverworts and 234 mosses) were collected. Twenty three taxa are reported here for the first time for Montenegro. Eight of the species recorded are redlisted in Europe.
\end{abstract}

Key words: liverworts, mosses, new national records, red listed species

Beáta Papp, Botanical Department, Hungarian Natural History Museum, PO Box 222, H-1476, Budapest, Hungary; e-mail: pappbea@bot.nhmus.hu

Peter Erzberger, Belziger Str. 37,D-10823 Berlin, Germany; e-mail: erzberger.peter@googlemail.com

Snežana Dragićević, Natural History Museum of Montenegro, Trg Bećir-bega Osmanagića 16, Podgorica 374, Montenegro; e-mail: sneza.dragicevic@t-com.me

\section{INTRODUCTION}

Exploration of the bryophyte flora of Montenegro is far from complete, although intensive fieldwork there in the last decade has yielded records of many new species. Knowledge about the bryophytes of Montenegro was first summarized in a checklist (Dragićević \& Veljić 2006), then in liverwort and moss checklists of Southeastern Europe and the Mediterranean (Ros et al. 2007, 2013; Sabovljević \& Natcheva 2006; Sabovljević et al. 2008). The liverwort checklist (Ros et al. 2007) gave 106 species from Montenegro. Later on some other works reported 32 more liverworts from the country (Erzberger \& Papp 2007; Erzberger et al. 2008; Papp \& Erzberger 2007a, 2008). The most recent moss checklist (Ros et al. 2013) gives 527 mosses recorded in Montenegro.

\footnotetext{
1 We are delighted to publish this paper in a volume honoring Tamás Pócs on his $80^{\text {th }}$ birthday. Our field trips with him in the Balkans are often recalled. Beáta Papp's first-ever collecting trip, with Tamás Pócs and his colleagues to Serbia in 1998 , began our series floristic investigations in the territory of former Yugoslavia, which have produced a huge amount of data and new regional reports on several species, making important contributions to European bryophyte checklisting and red-listing projects which continue today.
}

Two additional moss species were reported by Papp \& Erzberger (2011).

Here we publish the results of collecting trips in the Bjelasica Mts organized in 2007 and 2008 by the Hungarian Natural History Museum and the Natural History Museum of Montenegro. Erzberger et al. (2008) reported some taxa new to the bryophyte flora of Montenegro based on the 2007 fieldwork. The present paper contains the complete floristic results of both field trips and reports additional taxa new for the bryophyte flora of the country.

\section{MATERIAL AND METHODS}

\section{STUDY AREA}

The Bjelasica Mts (Fig. 1) form a high mountain range in the northern part of Montenegro, with several peaks reaching $\mathrm{ca} 2000 \mathrm{~m}$ a.s.l., such as Crna glava (2137), Zekova glava (2116), Troglav (2075), Jarčeve strane (2032) and Ogorela glava (1986). They are clearly separated from the neighboring Montenegrin mountains by deep and wide river valleys: from the Prokletije Mts in the east and southeast by the river Lim and from the Sinjavina and Morača Mts in the west by the river Tara. 
The relief of Bjelasica is diverse. There are many springs, streams, and seven glacial lakes situated between 1000 and $2000 \mathrm{~m}$ a.s.l. The area's geology is also very diverse. Volcanic and siliceous rocks are characteristic, but limestone, sandstone and slate can also be found (Lakušić 1966).

The climate of Bjelasica Mts is influenced by the Mediterranean, especially on the southern slopes; it is more continental in the northern parts, and montane climate prevails at higher altitudes. In the town of Kolašin lying at $1300 \mathrm{~m}$ a.s.l. the average annual temperature is $7.3^{\circ} \mathrm{C}$, the coldest month is January at $-1.9^{\circ} \mathrm{C}$, and the warmest month is July at $16^{\circ} \mathrm{C}$. The annual average of sunny hours is 1830 . Annual mean precipitation is $2016 \mathrm{~mm}$. The most humid month is December, while July is the driest (Radović \& Medenica 2006).

Biogradska gora National Park, situated in the central part of this massif, preserves a very impressive virgin forest with more than 86 tree species. It has many endemic plants and 11 habitats of Appendix I of the Bern Convention, for which it was designated an Important Plant Area (IPA) and an Emerald site (Kasom \& Hadžiablahović 2008).

\section{SITE DETAILS}

1. Bjelasica Mts, at Svinjača stream at Jezerine at Kolašin town, $1360 \mathrm{~m}, 42^{\circ} 49^{\prime} 49,5^{\prime \prime} \mathrm{N}, 19^{\circ} 37^{\prime} 06,7^{\prime \prime} \mathrm{E}$, 18 June 2007.

2. Bjelasica Mts, Katun Rupe at Kolašin town, 1660 m, 42 ${ }^{\circ} 49^{\prime} 09,9^{\prime \prime} \mathrm{N}, 19^{\circ} 36^{\prime} 59,7^{\prime \prime} \mathrm{E}, 19$ June 2007.

3. Bjelasica Mts, Bikovača between Ključ and Kapela at Kolašin town, $1880 \mathrm{~m}, 42^{\circ} 48^{\prime} 42,1^{\prime \prime} \mathrm{N}, 19^{\circ} 36^{\prime} 57,8^{\prime \prime} \mathrm{E}$, 19 June 2007.

4. Bjelasica Mts, above Kolašin town, $1360 \mathrm{~m}$, $42^{\circ} 49^{\prime} 13,7^{\prime \prime} \mathrm{N}, 19^{\circ} 34^{\prime} 20,6^{\prime \prime} \mathrm{E}, 19$ June 2007.

5. Bjelasica Mts, along a small side branch at Svinjača stream at Kolašin town, $1355 \mathrm{~m}, 42^{\circ} 50^{\prime} 18,2^{\prime \prime} \mathrm{N}$, 19³4'13,1"E, 20 June 2007.

6. Bjelasica Mts, Svinjača stream at Kolašin town, $1355 \mathrm{~m}, 42^{\circ} 50^{\prime} 18,2^{\prime \prime} \mathrm{N}, 19^{\circ} 34^{\prime} 13,1^{\prime \prime} \mathrm{E}, 20$ June 2007.

7. Bjelasica Mts, between Troglava and Zekova glava peaks at Kolašin town, $2022 \mathrm{~m}, 42^{\circ} 51^{\prime} 05,7^{\prime \prime} \mathrm{N}$, $19^{\circ} 40^{\prime} 08,8^{\prime \prime} \mathrm{E}, 20$ June 2007.

8. Bjelasica Mts, Zekova glava peak at Kolašin town, $2080 \mathrm{~m}, 42^{\circ} 51^{\prime} 04,2^{\prime \prime} \mathrm{N}, 1^{\circ} 40^{\prime} 54,6^{\prime \prime} \mathrm{E}, 20$ June 2007.

9. Bjelasica Mts, Biogradsko jezero lake at Mojkovac town, $1100 \mathrm{~m}, 42^{\circ} 54^{\prime} 02,6^{\prime \prime} \mathrm{N}, 19^{\circ} 35^{\prime} 45,6^{\prime \prime} \mathrm{E}$, 23 June 2008.

10. Bjelasica Mts, Biogradsko jezero lake at Mojkovac town, Alnetum at inflow of Biogradska reka stream, $1120 \mathrm{~m}, 42^{\circ} 53^{\prime} 44,3^{\prime \prime} \mathrm{N}, 19^{\circ} 36^{\prime} 14,7^{\prime \prime} \mathrm{E}, 23$ June 2008.

11. Bjelasica Mts, Jaževi at Kolašin town, western source of Biogradska reka stream, $1870 \mathrm{~m}, 42^{\circ} 51^{\prime} 03,9^{\prime \prime} \mathrm{N}$, $19^{\circ} 39^{\prime} 37,9^{\prime \prime}$ E, 25 June 2008.

12. Bjelasica Mts, source area of Biogradska reka stream (Izvor Biogradske reke) at Kolašin town, $1880 \mathrm{~m}$, $42^{\circ} 51^{\prime} 20,9^{\prime \prime} \mathrm{N}, 19^{\circ} 40^{\prime} 29,8^{\prime \prime} \mathrm{E}, 25$ June 2008.

13. Bjelasica Mts, towards Crna glava peak at Kolašin town, $1920 \mathrm{~m}, 42^{\circ} 52^{\prime} 22,8^{\prime \prime} \mathrm{N}, 19^{\circ} 40^{\prime} 42,9^{\prime \prime} \mathrm{E}$, 26 June 2008.

14. Bjelasica Mts, Crna glava peak at Kolašin town, $2070-2140 \mathrm{~m}, 42^{\circ} 52^{\prime} 03,2^{\prime \prime} \mathrm{N}, 19^{\circ} 41^{\prime} 24,2^{\prime \prime} \mathrm{E}$ and $42^{\circ} 51^{\prime} 59,6^{\prime \prime} \mathrm{N}, 19^{\circ} 41^{\prime} 53,1^{\prime \prime} \mathrm{E}, 26$ June 2008.

15. Bjelasica Mts, between Crna glava peak and Ursulovac lake at Kolašin town, 2060 m, 42 $52^{\prime} 03,2^{\prime \prime} \mathrm{N}$, $19^{\circ} 41^{\prime} 24,2^{\prime \prime}$ E, 26 June 2008.

16. Bjelasica Mts, around Ursulovac lake at Kolašin town, $2040-1920 \mathrm{~m}, 42^{\circ} 52^{\prime} 18,3^{\prime \prime} \mathrm{N}, 19^{\circ} 41^{\prime} 43,8^{\prime \prime} \mathrm{E}$ and $42^{\circ} 52^{\prime} 21,0^{\prime \prime} \mathrm{N}, 19^{\circ} 41^{\prime} 39,7^{\prime \prime} \mathrm{E}, 26$ June 2008.

17. Bjelasica Mts, between Ursulovac and Mali Ursulovac lakes, $1900 \mathrm{~m}, 42^{\circ} 52^{\prime} 26,4^{\prime \prime} \mathrm{N}, 19^{\circ} 41^{\prime} 32,9^{\prime \prime} \mathrm{E}$, 26 June 2008.

18. Bjelasica Mts, above Mali Ursulovac lake, $1830 \mathrm{~m}, 42^{\circ} 52^{\prime} 27,6^{\prime \prime} \mathrm{N}, 19^{\circ} 41^{\prime} 16,6^{\prime \prime} \mathrm{E}, 26$ June 2008.

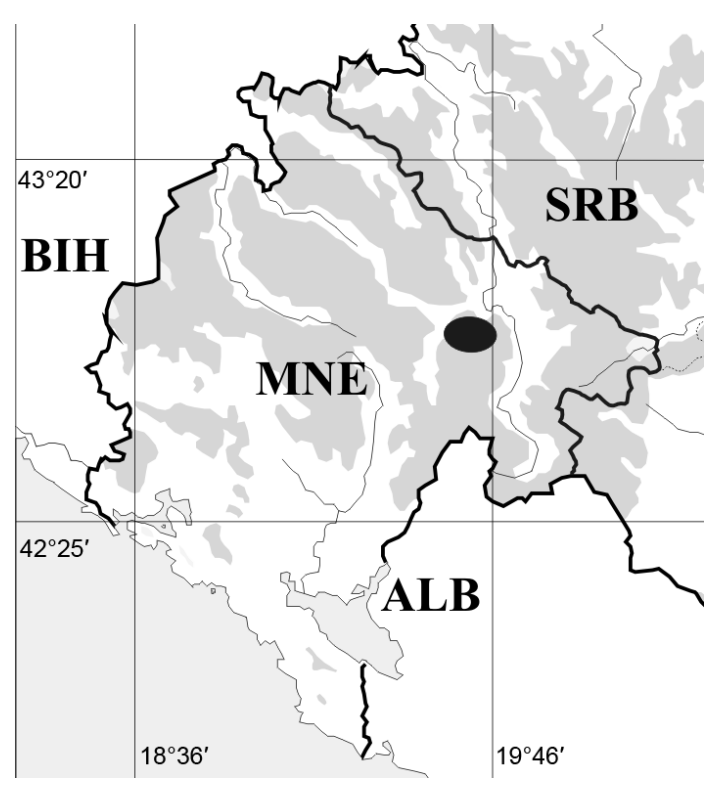

Fig. 1. Location of the investigated area. (BIH - BosniaHerzegovina, MNE - Montenegro, SRB - Serbia, ALB - Albania). 


\section{METHODS}

The collecting trips were undertaken in July 2007 and in July 2008. The investigated area is shown in Figure 1. All the main habitat types, such as stream valleys, springs, wet meadows, forests and alpine grasslands were investigated, and bryophytes were collected from different substrates (soil, exposed and shaded rocks, tree bark, decaying wood). Floristic results are considered new to Montenegro if there are no corresponding records in the bryophyte checklist of Montenegro (Dragićević \& Veljić 2006), the liverwort checklists of Southeastern Europe and the Mediterranean (Sabovljević \& Natcheva 2006; Ros et al. 2007), the moss checklist of Southeastern Europe (Sabovljević et al. 2008) and some other works containing additional data for Montenegro (Erzberger \& Papp 2007; Erzberger et al. 2008; Papp \& Erzberger 2007a, 2008).

The specimens were shared between the participating parties and are preserved in the Herbarium of the Hungarian Natural History Museum, Budapest (BP), and in the Herbarium of the Botanical Museum BerlinDahlem (B).

Nomenclature follows Grolle and Long (2000) for liverworts and Hill et al. (2006) for mosses, with the exception of Grimmia sessitana, which follows Maier (2002).

The distribution of new and interesting species in SE European countries is given according to the checklists of SE Europe (Sabovljević \& Natcheva 2006; Sabovljević et al. 2008) and the Mediterranean (Ros et al. 2007) unless otherwise cited.

For evaluation of the nature conservation status of species we used the following literature sources: the European Red Data Book of Bryophytes (Anonymous 1995), the bryophyte red list for Serbia and Montenegro (Sabovljević et al. 2004), the red list of bryophytes in Bulgaria (Natcheva et al. 2006), and the checklist and red list of the bryophytes of Romania (Ştefănuț \& Goia 2012). Comments on nature conservation status are given for species red-listed in Montenegro or in at least two other SE European countries. The various red lists differ slightly with respect to threat categories, conservation status and symbols. For the European Red Data Book of Bryophytes (Anonymous 1995) the symbols are as follows: Ex - Extinct, Ev - Vanished, E - Endangered, $\mathrm{V}$ - Vulnerable, $\mathrm{R}$ - Rare, $\mathrm{K}$ - Insufficiently known, $\mathrm{T}$ Taxonomically ill-defined taxa, RT - Regionally threatened species, and NT - Not threatened. The bryophyte red list for Serbia and Montenegro (Sabovljević et al. 2004) uses the following: EX - Extinct, CR - Critically endangered, EN - Endangered, VU - Vulnerable, LR -
Lower risk or near threatened, and DD - Data deficient. The red lists of the bryophytes of Bulgaria (Natcheva et al. 2006) and Romania (Ştefănut \& Goia 2012) use these symbols: CR - Critically endangered, EN - Endangered, VU - Vulnerable, NT - Near threatened, DD Insufficiently known, and NE - Not evaluated.

\section{RESULTS}

During our fieldwork we collected 318 bryophyte taxa (84 liverworts and 234 mosses), 23 of which are first records for the Montenegro (asterisked). The numerals following the species names refer to the collection sites described above.

\section{Hepaticae}

\section{"Anastrophyllum minutum (Schreb.)}

R. M. Schust.

COLLECTING SITES: 7, 11 - siliceous rock.

NotES. Boreal, montane species (Düll 1983). In SE Europe known only from Bulgaria, Croatia, Romania, Serbia, Slovenia. Threat status: LR in Serbia.

Anthelia juratzkana (Limpr.) Trevis.

COLLECTING SITES: 8,13 - soil among siliceous rock outcrops.

NOTES. Reported as new for the Montenegrin bryophyte flora based on part of this collection (site 8: Erzberger et al. 2008). In SE Europe known only from Bulgaria, Croatia, Former Yugoslav Republic of Macedonia, Romania, Slovenia. Threat status: VU in Bulgaria.

Apometzgeria pubescens (Schrank) Kuwah.

COLLECTING SITES: 5 - limestone rock and bark of Fagus; 9 - limestone rock.

Athalamia hyalina (Sommerf.) S. Hatt.

COLLECTING SITES: 8 - limestone rock; 11 - schistose rock; 14 - soil among limestone rocks.

NOTES. From Montenegro there are two old records from the Orjen Mts collected in the beginning of the last century (Schiffner 1916a, b; 
Dragićević \& Veljić 2006). Threat status: CR in Montenegro, VU in Bulgaria.

Barbilophozia barbata (Schreb.) Loeske

COLLECTING SITE: 9 - limestone rock.

Notes. Reported recently for the first time in Montenegro (Erzberger \& Papp 2007); known from several localities from the Durmitor Mts and Lovćen Mts (Papp \& Erzberger 2007a, 2010).

Barbilophozia hatcheri (A. Evans) Loeske

COLLECTING SITES: 1 - soil; 2, 7, 8 - siliceous rock; 11 - schistose rock.

Notes. Red-listed (LR) in Montenegro and Serbia, although apparently not rare in these countries as evidenced by several collections (Papp \& Erzberger 2007b, 2009; Papp et al. 2008, 2010, 2012).

\section{Barbilophozia lycopodioides (Wallr.) Loeske}

COLleCting SITES: 2, 7 - soil among siliceous rock outcrops; 11 - schistose rock; 14 - soil among limestone rocks.

\section{Blepharostoma trichophyllum (L.) Dumort.}

Collecting Sites: 1, 9 - decaying wood; 8 - soil among siliceous rock outcrops.

\section{Calypogeia azurea Stotler et Crotz}

COLLECTING SITES: 9 - soil; 12 - wet meadow.

Notes. According to Dragićević and Veljić (2006) known only from a very old record (Beck \& Szyszyłowicz 1888).

"Calypogeia muelleriana (Schiffn.) Müll. Frib.

COLLECTING SITES: 11 - schistose rock at rivulet; 12 wet meadow.

Notes. Subboreal montane species (Düll 1983). In SE Europe known only from Bulgaria, Romania, Serbia, Slovenia and the European part of Turkey. Recently reported from Albania (Marka \& Sabovljević 2011), Former Yugoslav Republic of Macedonia (Papp \& Erzberger 2012) and
Croatia (Papp et al. 2013). Threat status: CR in Serbia.

Calypogeia suecica (Arnell \& J. Perss.) Müll. Frib.

COLLECTING SITE: 1 - decaying wood.

NoTES. Reported as a new species for the Montenegrin bryophyte flora based on this collection (Erzberger et al. 2008); another record is from the Durmitor Mts (Papp \& Erzberger 2010). Threat status: EN in Serbia, VU in Bulgaria.

Cephalozia bicuspidata (L.) Dumort.

COllecting SITES: 1 - decaying wood; 12 - peat in spring mire; 16,18 - soil.

Cephalozia catenulata (Huebener) Lindb.

COLLECTING SITE: 9 - decaying wood.

NoTES. Recently reported for the first time in Montenegro from the Durmitor Mts (Papp \& Erzberger 2010). This is the second record for the country. Threat status: VU in Serbia, EN in Bulgaria.

Cephalozia connivens (Dicks.) Lindb.

COLLECTING SITE: 1 - decaying wood.

Cephaloziella divaricata (Sm.) Schiffn.

COLLECTING SITES: 2 - siliceous rock; 3 - limestone rock.

NOTES. Reported recently for the first time in Montenegro (Erzberger \& Papp 2007); known up to now from the Durmitor Mts, Rumija Mts and Kotor bay (Papp \& Erzberger 2007a, 2010).

*Cephaloziella rubella (Nees) Warnst.

COLLeCTING SITE: 12 - soil.

NotES. Northern subAtlantic species (Düll 1983). Recently reported from Albania (Marka \& Sabovljević 2011) and Greece (Papp et al. 2011). Known from almost all SE European countries except Bosnia-Herzegovina, FYR Macedonia 
and Montenegro. Threat status: VU in Serbia, EN in Bulgaria.

Chiloscyphus pallescens (Ehrh. ex Hoffm.)

Dumort.

COLLECTING SITES: $1,11,16$ - soil near spring.

\section{Chiloscyphus polyanthus (L.) Corda}

COLLECTING SITES: 1 - soil near stream; 2 - at spring; 12 - peat in spring mire.

Cololejeunea calcarea (Lib.) Schiffn.

COLLECTING SITE: 5 - limestone rock.

Conocephalum salebrosum Szweykowski,

Buczkowska \& Odrzykoski

COLLECTING SITES: 1,10 - soil at stream.

NOTES. Reported recently for the first time in Montenegro from the Durmitor Mts (Papp \& Erzberger 2010) where it was collected at two sites.

"Diplophyllum albicans (L.) Dumort.

COLleCting SITE: 11 - in dry rock crevice.

Notes. Northern sub-Atlantic species (Düll 1983). Known from almost all SE European countries except Albania, Montenegro and the European part of Turkey.

Diplophyllum taxifolium (Wahlenb.) Dumort.

COLLECTING SITE: 7 - siliceous rock (det. J. Váňa).

Notes. Reported as a new species for the Montenegrin bryophyte flora based on this collection (Erzberger et al. 2008). In SE Europe known only from Bulgaria, Romania and Slovenia.

Frullania dilatata (L.) Dumort.

COLLECTING SITES: 1 - bark of Abies; 2, 5 - bark of Fagus; 9 - limestone rock and bark of Fagus; 10 - bark of Alnus.

Frullania tamarisci (L.) Dumort.

COLLECTING SITES: 9 - bark of Fagus; 10 - bark of Alnus; 11 - limestone rock.
Gymnomitrion concinnatum (Lightf.) Corda

COLLECTING SITES: 7, 8 - siliceous rock; 11 - schistose rock.

NotES. Reported as a new species for the Montenegrin bryophyte flora based on part of this collection (site 8: Erzberger et al. 2008). In SE Europe known only from Bulgaria, Romania and Slovenia.

Harpanthus scutatus (F. Weber \& D. Mohr) Spruce

COLLECTING SITE: 10 - decaying wood.

Notes. According to Dragićević and Veljić (2006) there was only a very old doubtful record from Montenegro in Beck and Szyszyłowicz (1888). In SE Europe known only from Bulgaria, Bosnia-Herzegovina, Croatia, Romania, Slovenia and the European part of Turkey. Threat status: DD in Bulgaria.

\section{Jamesoniella autumnalis (DC.) Steph.}

COLleCting SITE: 11 - schistose rock (conf. S. Stefanuț).

Notes. According to Dragićević and Veljić (2006) there was only a very old doubtful record from Montenegro (Beck \& Szyszyłowicz 1888).

\section{Jungermannia confertissima Nees}

COLleCting SITE: 16 - soil (conf. S. Stefanuț).

NoTES. Recently reported for the first time in Montenegro from the Durmitor Mts (Papp \& Erzberger 2010) where it was found at two sites. This is the third record for the country. In SE Europe known only from Bulgaria, Romania and Slovenia. Threat status: VU in Bulgaria.

\section{Jungermannia gracillima $\mathrm{Sm}$.}

COLlecting SITE: 14 - soil (conf. S. Stefanuț).

NoTES. Reported recently for the first time in Montenegro from the Durmitor Mts (Erzberger \& Papp 2007). This is the second record for the country. 


\section{*Jungermannia hyalina Lyell}

Collecting Site: 16 - soil (conf. S. Stefanuț and J. Váňa).

Notes. This species of mountain areas of the temperate zone of Europe (Düll 1983) is known from almost all SE European countries except Montenegro and FYR Macedonia, but has recently been reported from FYR Macedonia also (Papp \& Erzberger 2012).

\section{Jungermannia sphaerocarpa Hook.}

COLLECTING SITES: 13 - soil near late snow bed; 18 peat in spring mire (det. J. Váňa).

Leiocolea bantriensis (Hook.) Jörg.

COLLECTING SITE: 16 - soil.

Notes. Threat status: VU in Serbia.

\section{Leiocolea collaris (Nees) Schljakov}

COLLECTING SITES: 2, 8 - limestone rock.

Notes. Red-listed (VU) in Montenegro, although apparently not rare there as evidenced by several records (Papp \& Erzberger 2007a, 2010, Papp et al. 2008).

\section{Leiocolea heterocolpos (Hartm.) H. Buch}

COLLECTING SITES: 11, 14 - soil among limestone rocks; 16 - soil.

Notes. In the Durmitor Mts found in several localities (Papp \& Erzberger 2010). Threat status: VU in Montenegro.

Lejeunea cavifolia (Ehrh.) Lindb.

COLlecting Sites: 2 -siliceous rock; 10 - bark of Alnus.

Lepidozia reptans (L.) Dumort.

COLLECTING SITES: 1, 9 - decaying wood.

Lophocolea heterophylla (Schrad.) Dumort.

COLLECTING SITES: 1, 9 - decaying wood; 14 - soil.

\section{Lophocolea minor Nees}

COLLECTING SITE: 16 - soil at lake shore.

Lophozia ascendens (Warnst.) R. M. Schust.

COLLECTING SITE: 1 - decaying wood.

Notes. Reported as a new species for the Montenegrin bryophyte flora based on this collection (Erzberger et al. 2008); also known from the Durmitor Mts (Papp \& Erzberger 2010). Threat status: $\mathrm{R}$ in the Red Data Book of European Bryophytes, VU in Bulgaria, Serbia and Romania.

Lophozia excisa (Dicks.) Dumort.

COLLECTING SITE: 3 - soil among limestone rocks.

Lophozia incisa (Schrad.) Dumort.

COLleCting SITES: 1 - decaying wood; 8 - siliceous rock; 15 - acidic soil.

NOTES. Reported recently for the first time in Montenegro from the Durmitor Mts (Erzberger \& Papp 2007) where it is known from several localities (Papp \& Erzberger 2010).

Lophozia longidens (Lindb.) Macoun

COLLECTING SITE: 11 - schistose rock (det. J. Váňa).

NOTES. Reported recently for the first time in Montenegro from the Durmitor Mts (Papp \& Erzberger 2010) where it is known from two localities.

Lophozia longiflora (Nees) Schiffn.

Collecting SITE: 1 - decaying wood (conf. S. Stefanuț).

Lophozia obtusa (Lindb.) A. Evans

Collecting SITE: 1 - soil (det. B. Cykowska).

NoTES. Reported recently for the first time in Montenegro from the Durmitor Mts (Papp \& Erzberger 2010) where it is known from several localities. 
"Lophozia opacifolia Culm. ex Meyl.

COLLECTING SITE: 16 - soil among siliceous rocks near lake (det. J. Váňa).

Notes. Among the SE European countries known only from Romania where it is designated EN.

\section{Lophozia sudetica (Nees ex Huebener) Grolle}

COLLECTING SITES: 2, 7, 12,16-siliceous rock; 8 - siliceous rock (conf. B. Cykowska, J. Váňa); 11 - schistose rock; 18 - soil.

Notes. Reported as new for the Montenegrin bryophyte flora partly based on this collection (Erzberger et al. 2008); apparently not rare in the Bjelasica Mts; also known from the Orjen Mts (Papp et al. 2008).

\section{Lophozia ventricosa (Dicks.) Dumort.}

COLLECTING SITES: 1 - decaying wood; 7, 8, 16 - siliceous rock; 11 - schistose rock.

\section{${ }^{*}$ Lophozia wenzelii (Nees) Steph.}

COLLeCtING SITES: 8,15 - acidic soil (conf. S. Stefanuț).

Notes. Boreal, montane element (Düll 1983). Known in SE Europe only from Bulgaria, Greece and Romania. Recently reported from FYR Macedonia (Papp et al. 2011).

Mannia androgyna (L.) A. Evans

COLLECTING SITE: 3 - soil among limestone rocks.

Notes. Threat status: CR in Montenegro and Bulgaria.

Marchantia polymorpha L. subsp. montivagans Bisch. \& Boisselier

Collecting Sites: 2 - at spring; $12,16,18$ - wet meadow.

\section{Marsupella funckii (F. Weber \& D. Mohr)}

Dumort.

COLleCting Sites: 7 (det. J. Váňa), 8 (conf. S. Stefanuț) - siliceous rock; 11 (conf. S. Stefanuț), 13 (det. J. Váňa),
17 (conf. S. Stefanuț) - schistose rock; 15 (conf. S. Stefanuț - acidic soil; 16 (conf. S. Stefanuț) - wet meadow.

NOTES. Reported recently for the first time in Montenegro from the Durmitor Mts (Papp \& Erzberger 2010) where it was found in one locality; apparently not rare in the Bjelasica Mts. Threat status: VU in Serbia and Bulgaria.

Marsupella sphacelata (Gieseke ex Lindenb.)

Dumort.

COLleCting SITES: 8 - siliceous rock (det. J. Váňa); 11 (conf. S. Stefanuț) - schistose rock; 18 (conf. S. Stefanuț) - wet meadow.

NOTES. Reported as a new species for the Montenegrin bryophyte flora based on part of this collection (site 8: Erzberger et al. 2008). In SE Europe reported only from Bulgaria, Greece, Romania and recently from FYR Macedonia (Papp et al. 2011). Threat status: NT in Romania.

Metzgeria conjugata Lindb.

COLLECTING SITES: 5, 9 - limestone rock.

Metzgeria furcata (L.) Dumort.

COLLECTING SITES: 1 - siliceous rock and decaying wood; 2 - siliceous rock; 5 - limestone rock; 10 - bark of Alnus.

\section{*Nardia scalaris Gray}

COLLECTING SITES: 13,18 - soil.

Notes. A species of mountain areas of the western temperate zone of Europe (Düll 1983). In SE Europe known only from Bulgaria, Croatia, Greece, Romania, Serbia, Slovenia and the European part of Turkey.

\section{Nowellia curvifolia (Dicks.) Mitt.}

COLLECTING SITES: 1, 9 - decaying wood.

NotES. Red-listed (VU) in Montenegro and Serbia, although apparently not rare in these countries as evidenced by numerous records (Papp \& Erzberger 2005, 2010, Papp \& Sabovljević 2002). 
Pedinophyllum interruptum (Nees) Kaal.

COLLECTING SITE: 5 - limestone rock.

Pellia endiviifolia (Dicks.) Dumort.

COLLECTING SITES: 1 - soil near stream; 5 - limestone rock in stream; 16 - wet meadow.

Pellia neesiana (Gottsche) Limpr.

COLLECTING SITES: 1 - at stream; 12 - wet meadow.

Notes. According to Dragićević and Veljić (2006) there was only a very old doubtful record from Montenegro (Beck \& Szyszyłowicz 1888).

Plagiochila asplenioides (L.) Dumort.

COLLECTING SITE: 5 - soil near stream.

\section{Plagiochila porelloides (Torrey ex Nees)}

Lindenb.

COlLeCting SITES: 1 - soil; 2 - siliceous rock, soil among basic rock outcrops; 5 - on bark of Fagus and limestone rock in stream; 7 - soil; 8 - soil among limestone and siliceous rocks; 9,14 - limestone rock; $10-$ bark of Alnus; 11 - soil near spring and schistose rock; 12 - soil near siliceous rock outcrops.

Porella baueri (Schiffn.) C. E. O. Jensen

COLLECTING SITE: 5 - limestone rock.

NOTES. From Montenegro also known from the Durmitor Mts (Papp \& Erzberger 2010). Threat status: DD in Serbia, NT in Bulgaria.

Porella cordaeana (Huebener) Mohr

COLLECTING SITES: 1 - soil; 2, 3, 12 - siliceous rock; 11 - soil near spring.

Porella platyphylla (L.) Pfeiff.

COLLECTING SITES: 2 - siliceous boulder; 5 - bark of Fagus.

\section{Preissia quadrata (Scop.) Nees}

COLLECTING SITES: 2,8 - limestone rock; 11 - schistose rock; 16 - soil.
Ptilidium pulcherrimum (Weber) Vain.

COLLECTING SITE: 1 - bark of Abies.

Radula complanata (L.) Dumort.

COLLECTING SITES: 1 - siliceous rock and bark of Abies; 2 - siliceous rock; 5 - bark of Fagus; 9 - base of Fagus; 10 - bark of Alnus; 11 - schistose rock at rivulet.

Radula cf. lindenbergiana Gottsche ex C. Hartm. COLLECTING SITE: 2 - siliceous boulder (det. J. Váňa).

Reboulia hemisphaerica (L.) Raddi

COLLECTING SITES: 8 - limestone rock; 11 - schistose rock.

Riccardia palmata (Hedw.) Carruth.

COLLECTING SITE: 1 - decaying wood.

Riccia sorocarpa Bisch.

COLleCting SITES: 2 - limestone rock; 3, 14 - soil among limestone rocks.

Scapania aequiloba (Schwägr.) Dumort.

COLLECTING SITES: 2, 14 - limestone rock; 11 - schistose rock.

Scapania aspera $\mathrm{M}$. Bernet \& Bernet

COLleCting Sites: 2, 5, 8, 9, 14 - limestone rock; 11 - schistose rock.

Scapania compacta (A. Roth) Dumort.

Collecting Sites: 9, 16 (det. J. Váňa) - soil.

NoTES. From Montenegro there is a very old record (Loitlesberger 1905; Dragićević \& Veljić 2006) and a recent one (Papp \& Erzberger 2007a) from the same area around Herceg Novi. Threat status: EN in Bulgaria, DD in Romania.

Scapania irrigua (Nees) Nees

COLLECTING SITES: 12 - wet meadow; 15 - acidic soil.

Scapania nemorea (L.) Grolle

COLlecting Sites: 1 (conf. J. Váňa), 9 - soil. 
"Scapania mucronata $\mathrm{H}$. Buch

COLLeCting SITE: 16 - soil (conf. S. Stefanuț).

NOTES. Subboreal, montane species (Düll 1983). In SE Europe known only from Bulgaria, Romania and Serbia.

"Scapania scandica (Arnell \& H. Buch)

Macvicar

Collecting Site: 1 (conf. S. Stefanuț, cf. det. J. Váňa), 16 (cf. det. J. Váňa) - soil.

NOTES. Subarctic, montane element (Düll 1983). Known in SE Europe only from Bulgaria, Romania and Serbia. Threat status: EN in Bulgaria, $\mathrm{VU}$ in Romania.

\section{"Scapania praetervisa Meyl.}

COLLECTING SITES: 3, 14 - soil among limestone rocks (conf. S. Stefanuț).

NoTES. Arctic, alpine element (Düll 1983). Not mentioned from any SE European country.

Scapania umbrosa (Schrad.) Dumort.

COLLECTING SITE: 1 - decaying wood.

Scapania undulata (L.) Dumort.

COLLECTING SITES: 1 - at stream; 11 - schistose rock at rivulet; 12 - siliceous rock at rivulet; 18 - peat in spring mire.

Scapania undulata (L.) Dumort. var. dentata (Dumort.) Jörg. Bergens

COLLECTING SITE: 18 - wet meadow.

Tritomaria exsecta (Schmidel) Loeske

COLLECTING SITES: 1,9 - decaying wood.

NoTES. According to Dragićević and Veljić (2006) there was only a very old doubtful record from Montenegro (Beck \& Szyszyłowicz 1888).

Tritomaria quinquedentata (Huds.) H. Buch

COLLECTING SITES: 1, 2 - soil; 14 - soil among limestone rocks.
NoTES. Reported as new for the Montenegrin bryophyte flora based on part of this collection (site 2: Erzberger et al. 2008).

\section{MusCI}

Abietinella abietina (Hedw.) M. Fleisch.

COLLECTING SITES: 1,2 - soil; 13 - soil among limestone rocks.

Amphidium mougeotii (Schimp.) Schimp.

COLLECTING SITE: 2 - siliceous rock.

Andreaea rupestris Hedw.

COLleCting SITES: 1, 2, 7, 8, 12 - siliceous rock; 11 schistose rock.

Anomodon attenuatus (Hedw.) Huebener

COLLeCtiNg SITES: 5, 9 - limestone rock and bark of Fagus.

Anomodon longifolius (Schleich. ex Brid.) Hartm.

COLLECTING SITE: 9 - limestone rock.

NoTES. Red-listed (DD) in Montenegro and Serbia, but recently several records from Serbia have been published (Papp et al. 2006; Papp \& Erzberger 2007b, 2009; Papp \& Sabovljević 2001).

Anomodon viticulosus (Hedw.) Hook. \& Taylor COLLECTING SITES: 5, 9 - limestone rock and bark of Fagus.

Antitrichia curtipendula (Hedw.) Brid.

COLLECTING SITES: 5 - limestone rock and bark of Fagus; 6 - limestone rock at stream; 9 - siliceous rock; 10 - bark of Alnus incana.

Atrichum undulatum (Hedw.) P. Beauv.

COLLECTING SITES: 1,9 - soil.

Aulacomnium palustre (Hedw.) Schwägr.

COLLECTING SITE: 12 - wet meadow. 
Barbula unguiculata Hedw.

COLLECTING SITE: 16 - soil.

Bartramia halleriana Hedw.

COLLECTING SITES: 9 - soil; 11 - schistose rock.

\section{Bartramia ithyphylla Brid.}

COLlecting SITES: 1,8 - soil; 2, 7 - siliceous rock; 11 - schistose rock; 15,16 - acidic soil.

Brachytheciastrum velutinum (Hedw.) Ignatov \& Huttunen

COLLECTING SITES: 1 - soil; 2 - siliceous rock; 9 - bark of Fagus; 14 - soil among limestone rocks.

Brachythecium albicans (Hedw.) Schimp.

COLLeCting SITES: 1, 13 - soil among siliceous and limestone rocks.

\section{Brachythecium geheebi Milde}

COLLECTING SITE: 2 - siliceous rock.

NOTES. There are four more recent records from Montenegro but three of them are from one mountain area, the Durmitor Mts (Dragićević \& Veljić 2006). Threat status: R in the Red Data Book of European Bryophytes, LR in Montenegro, $\mathrm{EN}$ in Bulgaria, CR in Romania.

\section{Brachythecium glareosum (Bruch ex Spruce) Schimp.}

COLLECTING SITES: 1 - soil, limestone and siliceous rock, decaying wood; 3, 9 - limestone rock; 14 - soil among limestone rocks.

\section{Brachythecium rivulare Schimp.}

COLLECTING SITES: 1,10 - soil at stream; 2 - at spring; 5, 6- limestone rock in and near stream; 11 - soil among schistose rock at rivulet; 12,16 - peat in spring mire.

\section{Brachythecium rutabulum (Hedw.) Schimp.}

COLLECTING SITES: 1 - soil; 9 - soil and siliceous rock, base of Fagus and decaying wood; 10 - soil at stream and on bark of Alnus.
Brachythecium salebrosum (Hoffm. ex F. Weber \& D. Mohr) Schimp.

COLLECTING SITE: 1 - limestone rock.

Brachythecium tommasinii (Sendtn. ex Boulay) Ignatov \& Huttunen

COLLECTING SITE: 9 - limestone rock.

\section{Bryoerythrophyllum recurvirostrum (Hedw.)}

P. C. Chen

Collecting Sites: 8, 9, 14, 16 - limestone rock.

Bryum argenteum Hedw.

COLLECTING SITES: 7 - siliceous rock; 13,14 - soil among limestone rocks.

\section{Bryum caespiticium Hedw.}

COllecting SITES: 7 - siliceous rock; 12 (conf. W. Schröder), 13 - soil among limestone rocks.

Bryum capillare Hedw.

COLLECTING SITES: 1 - soil and siliceous rock; 2,3 - soil near basic rock outcrops; 6 - limestone rock in stream; 9 - limestone rock.

\section{Bryum elegans Nees}

COLLECTING SITES: 2 - siliceous and limestone rock; 8,14 - limestone rock; 11 - soil near basic rock outcrops.

Bryum moravicum Podp.

COLLeCting SITES: 1, 2, 7 - siliceous rock and bark of Fagus.

Bryum pallens Sw. ex anon.

Collecting Sites: 2 - limestone rock (conf. W. Schröder); 11 - schistose rock at rivulet (conf. W. Schröder).

Bryum pallescens Schleich. ex Schwägr.

COLLECTING SITES: 3,8 - soil among limestone rocks; 11, 12 - soil near spring (det. W. Schröder). 
Bryum pseudotriquetrum (Hedw.) P. Gaertn. et al.

COLLECTING SITES: 1 - soil at stream; 11 - schistose rock at rivulet; $12,16,18$ - peat in spring mires; 15 at spring.

\section{Bryum rubens Mitt.}

COLLECTING SITE: 2 - soil.

\section{Bryum schleicheri DC.}

COLLECTING SITES: 2 - at spring; 11 - schistose rock at rivulet (also var. latifolium) (conf./rev. W. Schröder); 12 - wet meadow.

Buxbaumia viridis (Moug. ex Lam. \& DC.) Brid. ex Moug. \& Nestl.

COLLECTING SITES: 1, 9 - decaying wood.

Notes. Several extant populations are known in Montenegro (Dragićević et al. 2011). Threat status: listed in the Bern Convention and the European Union Habitats and Species Directives (Anonymous 1995), VU in the Red Data Book of European bryophytes, CR in Montenegro and Serbia, NT in Bulgaria, EN in Romania.

\section{Calliergonella cuspidata (Hedw.) Loeske}

COLLECTING SITES: 4,12 - peat in wet meadow.

\section{Campyliadelphus chrysophyllus (Brid.)}

R. S. Chopra

COLLECTING SITE: 8 - limestone rock.

\section{Campylium protensum (Brid.) Kindb.}

COLLECTING SITES: 11,12 - soil and peat near springs.

NotES. Probably overlooked, not separated from $C$. stellatum. From Montenegro there are several records from the Durmitor Mts (Papp \& Ezberger 2010). Threat status: VU in Bulgaria, $\mathrm{EN}$ in Romania.

\section{Campylium stellatum (Hedw.) Lange}

\& C. E. O. Jensen

COLleCting SITES: 11 - schistose rock at rivulet; 12, 16 - wet meadow.
Campylophyllum halleri (Hedw.) M. Fleisch.

COLLECTING SITES: 1 - siliceous rock; 14 - limestone rock.

Ceratodon purpureus (Hedw.) Brid.

COLleCting SITES: 1 - soil; 2 - siliceous rock and bark of Fagus; 7 - siliceous rock; 11 - schistose rock; 13 - soil among limestone rocks.

Cinclidotus aquaticus (Hedw.) Bruch \& Schimp.

COLLECTING SITE: 5 - limestone rock in stream.

Cinclidotus fontinaloides (Hedw.) P. Beauv.

COLLECTING SITE: 6 - limestone rock in stream.

Cirriphyllum crassinervium (Taylor) Loeske $\&$ M. Fleisch.

COLleCting SITES: 5, 9 - limestone rock.

Cirriphyllum piliferum (Hedw.) Grout

COLLECTING SITE: 10 - bark of Alnus.

Climacium dendroides (Hedw.) F. Weber

\& D. Mohr

COLLECTING SITE: 5 - soil near stream.

Cratoneuron filicinum (Hedw.) Spruce

COLLECTING SITES: 1 - at stream; 4 - soil in wet meadow; 5 - shaded limestone rock and limestone rock in stream; 11 - schistose rock at rivulet; 15 - at spring.

Ctenidium molluscum (Hedw.) Mitt.

COLLECTING SITES: 1, 2 - siliceous and limestone rock; $5,9,14$ - limestone rock.

"Dichodontium palustre (Dicks.) M. Stech

COLleCting SITES: 12,18 - peat in spring mires.

NOTES. Boreal, montane element (Düll 1984). Known from almost all SE European countries except Albania, Bosnia-Herzegovina, Montenegro and the European part of Turkey. Threat status: LR in Serbia. 
Dichodontium pellucidum (Hedw.) Schimp.

COLLECTING SITES: 1 - at stream; 11 - schistose rock at rivulet; 12,16 - wet meadow; 13 - soil near late snow bed; 15 - at spring.

Dicranella heteromalla (Hedw.) Schimp.

COLLECTING SITES: 7 - siliceous rock; 9 - soil; 11 schistose rock.

Dicranella varia (Hedw.) Schimp.

COLLECTING SITE: 1 - soil.

Dicranoweisia crispula (Hedw.) Milde

COLleCting SITES: $1,2,7,8,12,13,17$ - siliceous rock; 11 - schistose rock.

Dicranum scoparium Hedw.

COLlECTING SITES: 1 - decaying wood; 2, 5, 9 - limestone and other basic rock; 7, 8 - siliceous rock; 11 schistose rock; 13 - soil among limestone rocks.

Dicranum spadiceum J. E. Zetterst.

COLLECTING SITE: 11 - schistose rock.

NoTES. Reported recently for the first time in Montenegro from the Durmitor Mts (Papp \& Erzberger 2010). This is its second record from the country. In SE Europe known only from Bulgaria, Romania, Serbia and Slovenia.

\section{Dicranum tauricum Sapjegin}

COLLECTING SITE: 1 - bark of Abies and decaying wood.

Didymodon fallax (Hedw.) R. H. Zander

COLLECTING SITES: 2, 3 - limestone rock.

Didymodon rigidulus Hedw.

COLLECTING SITES: 1 - siliceous rock; 2, 5 - limestone and other basic rock; 6 - limestone rock in stream.

Diphyscium foliosum (Hedw.) D. Mohr

COLLECTING SITE: 9 - soil.

Notes. Red-listed (LR) in Montenegro and Serbia, but numerous records from these countries
(Papp et al. 2007b; Papp \& Erzberger 2005) and apparently frequent in some areas, e.g., around Vlasina lake in Serbia (Papp et al. 2012).

Distichium capillaceum (Hedw.) Bruch \& Schimp.

COLleCting Sites: $3,8,12,14$ - limestone and other basic rock; 11 - schistose rock at rivulet.

Ditrichum flexicaule (Schwägr.) Hampe

COLLECTING SITES: 2, 14 - limestone rock.

Ditrichum gracile (Mitt.) Kuntze

COLLECTING SITE: 2 - limestone rock.

Ditrichum heteromallum (Hedw.) E. Britton

COLLECTING SITE: 15 - acidic soil.

Ditrichum pusillum (Hedw.) Hampe

COLLECTING SITE: 1 - soil.

Notes. Threat status: LR in Montenegro. Probably overlooked and under-collected. Usually present in our recent collections (Papp \& Erzberger 2007a, 2010).

\section{Encalypta alpina $\mathrm{Sm}$.}

COLLECTING SITE: 14 - limestone rock.

Threat status: LR in Montenegro and Serbia, VU in Romania. From Montenegro there are two old records and a recent one from the Prokletije Mts (Dragićević \& Veljić 2006).

Encalypta ciliata Hedw.

COLLECTING SITE: 2 - limestone rock.

Threat status: VU in Montenegro and Serbia. In Montenegro it was also collected in the Durmitor Mts (Papp \& Erzberger 2010).

Encalypta microstoma Bals.-Criv. \& De Not.

COLLECTING SITE: 7 - siliceous rock.

NOTES. Reported as a new species for the Montenegrin bryophyte flora based on this collection 
(Erzberger et al. 2008). Threat status: R in the Red Data Book of European Bryophytes, VU in Bulgaria.

\section{Encalypta rhaptocarpa Schwägr.}

COLlecting SITE: 3 - limestone rock.

\section{Encalypta streptocarpa Hedw.}

COLLECTING SITES: 1 - concrete; 9, 14 - limestone rock; 11 - schistose rock.

\section{Encalypta vulgaris Hedw.}

COLLECTING SITES: 8, 14 - limestone rock.

Eurhynchiastrum pulchellum (Hedw.) Ignatov \& Huttunen var. diversifolium (Schimp.) Ochyra \& Żarnowiec

Collecting SITES: 2, 3, 13, 14 - soil among limestone and other basic rocks; 8 - limestone rock.

\section{Eurhynchium angustirete (Broth.) T. J. Kop.}

COLLECTING SITES: 1 - soil; 9 - limestone rock.

Fissidens bryoides Hedw.

COLLECTING SITES: 1 - soil; 11,14 - soil among limestone and other rocks; 16 - soil at lake shore.

Fissidens dubius P. Beauv.

COLlecting SITES: 8, 9, 14 - limestone rock.

Fissidens taxifolius Hedw.

COLlecting SITES: 1,9 - soil.

\section{Fontinalis antipyretica Hedw.}

COLLECTING SITES: 5, 6 - limestone rock in stream; 10 - soil at stream.

Notes. Red-listed (LR) in Montenegro and Serbia. This probably needs to be amended, since we collected this species in a great number of places in these countries (Papp et al. 2004; 2006, 2012; Papp \& Erzberger 2005, 2007a, b, 2009; Papp \& Sabovljević 2001).
Funaria hygrometrica Hedw.

COLLECTING SITE: 2 - soil.

${ }^{*}$ Grimmia alpestris (F. Weber \& D. Mohr) Schleich.

COLleCting SITES: 2, 12, 16 - siliceous rock (conf./ rev. E. Maier in part).

Notes. This is a boreal montane element (Düll 1984) known from almost all SE European countries except Bosnia-Herzegovina, Croatia, Montenegro, Slovenia and the European part of Turkey (Sabovljević et al. 2008).

Grimmia anomala Hampe ex Schimp.

COLLECTING SITES: 2, 16 - siliceous rock; 11 - schistose rock at rivulet (conf. E. Maier in part).

Notes. Reported as new for the Montenegrin bryophyte flora based on part of this collection (Erzberger et al. 2008).

Grimmia caespiticia (Brid.) Jur.

COLleCting SITES: 7, 12, 16, 17 - siliceous rock (in part conf. E. Maier).

Notes. In Montenegro also known from the Prokletije Mts (Martinčič 2006). Threat status: R in the Red Data Book of European Bryophytes, VU in Montenegro, CR in Romania.

Grimmia decipiens (Schultz) Lindb.

COLLECTING SITE: 12 - siliceous rock near spring.

NOTES. From Montenegro there are records from the Prokletije Mts (Martinčič 2006) and Morača gorge (Milikić et al. 2001). Threat status: $\mathrm{VU}$ in Bulgaria, EN in Romania.

Grimmia funalis (Schwägr.) Bruch \& Schimp.

COLLECTING SITE: 7 - siliceous rock (det./conf. E. Maier in part).

Notes. In Montenegro also known from the Prokletije Mts (Martinčič 2006). Threat status: VU in Bulgaria, NT in Romania. 


\section{Grimmia hartmannii Schimp.}

Collecting Sites: 2, 7, 9, 10 - siliceous rock (in part conf. E. Maier).

Notes. Grimmia hartmanii was found with sporophytes at site 2; sporophytes are extremely rare in this species (Nebel \& Philippi 2000).

Grimmia montana Bruch \& Schimp.

COLLECTING SITES: 2,7 - siliceous rock (in part conf. E. Maier).

NOTES. In Montenegro also known from the Prokletije Mts (Martinčič 2006). This is a widespread Grimmia species on exposed acidic rocks in neighboring countries, e.g., Stara planina and around Vlasina lake in Serbia (Papp \& Erzberger 2007b; Papp et al. 2012), the Korça region in Albania (Papp et al. 2010), and FYR Macedonia (Papp et al. 2011; Papp \& Erzberger 2012). Threat status: VU in Bulgaria, NT in Romania.

\section{Grimmia muehlenbeckii Schimp.}

COLLECTING SITES: 1, 2, 12 - siliceous rock; 11 - schistose rock at rivulet (in part conf. E. Maier).

NoTES. Reported recently for the first time in Montenegro from the Durmitor Mts (Papp \& Erzberger 2010) where it was found in one locality. Apparently not rare in the Bjelasica Mts. (Erzberger et al. 2008). Threat status: VU in Bulgaria and Romania.

This is one of the most frequent Grimmia species on exposed acidic rocks in neighboring countries, e.g., the Golija Biosphere Reserve, Stara planina and around Vlasina lake in Serbia (Papp \& Erzberger 2005, 2007b; Papp et al. 2012), the Korça region in Albania (Papp et al. 2010), and FYR Macedonia (Papp et al. 2011; Papp \& Erzberger 2012).

Grimmia ovalis (Hedw.) Lindb.

COLLECTING SITES: 2,3 - siliceous rock (in part conf. E. Maier).

Grimmia ramondii (Lam. \& DC.) Margad.

COLlECTING SITES: 1, 2, 7 - siliceous rock; 11 - schistose rock; 12 - wet meadow (in part conf. E. Maier).
Notes. In Montenegro also known from the Prokletije Mts (Martinčič 2006). Apparently not rare in the Bjelasica Mts. Threat status: VU in Bulgaria, EN in Romania.

\section{*Grimmia sessitana De Not.}

COLLECTING SITE: 11 - schistose rock.

Notes. Subarctic, subalpine element (Düll 1984); was known only from Bulgaria, FYR Macedonia, Romania and Serbia (Sabovljević et al. 2008). Recently also reported from Albania (Papp et al. 2010) and Greece (Papp et al. 2011). Threat status: R in the Red Data Book of European Bryophytes, EN in Romania.

\section{Grimmia torquata Drumm.}

COLLECTING SITE: 2 - siliceous rock.

NOTES. Reported as new for the Montenegrin bryophyte flora based on this collection (Erzberger et al. 2008). Threat status: VU in Bulgaria, EN in Romania. In Montenegro this is the only record so far.

${ }^{*}$ Hedwigia ciliata (Hedw.) P. Beauv. var. leucophaea Bruch \& Schimp.

COLLECTING SITE: 2 - siliceous rock.

Not reported at infrataxon level from Montenegro even in Düll et al. (1999). Known from Albania (Marka \& Xhulaj 2011), FYR Macedonia (Martinčič 2009), Greece (Düll 1995) and Slovenia (Martinčič 2003).

Herzogiella seligeri (Brid.) Z. Iwats.

COLLECTING SITES: 1, 9 - decaying wood.

Heterocladium dimorphum (Brid.) Schimp.

COLLECTING SITES: 2, 7 - soil among siliceous and basic rocks; 8 - soil among siliceous and limestone rock; 11 schistose rock, 14 - soil among limestone rocks.

Homalia trichomanoides (Hedw.) Brid.

COLLeCTING SITE: 10 - bark of Alnus. 
Homalothecium lutescens (Hedw.) H. Rob.

COLLECTING SITE: 1 - soil.

Homalothecium philippeanum (Spruce) Schimp.

COLLECTING SITES: 2 - siliceous boulder; 5 - limestone rock; 9 - limestone rock and bark of Fagus.

Homalothecium sericeum (Hedw.) Schimp.

COLLECTING SITES: 2 - siliceous boulder; 5 - basic rock and bark of Fagus and Sambucus nigra; 9 - limestone and siliceous rock; 10 - basic rock and bark of Alnus.

Homomallium incurvatum (Schrad. ex Brid.)

Loeske

COLLECTING SITE: 9 - limestone rock.

\section{Hygrohypnum duriusculum (De Not.)}

D. W. Jamieson

COLLECTING SITES: 2 - at spring; 12 - wet meadow.

Hygrohypnum luridum (Hedw.) Jenn.

COLLECTING SITES: 1 - at stream; 5, 6-limestone rock in stream.

Hylocomium splendens (Hedw.) Schimp.

COLleCting SITES: 1 - soil; 3, 5, 9 - limestone rock.

"Hypnum andoi A. J. E. Sm.

COLLECTING SITE: 1 - siliceous rock.

Notes. Atlantic element (Düll 1985). Known from Bosnia-Herzegovina, Greece, Romania, Serbia, Slovenia (Sabovljević et al. 2008) and FYR Macedonia (Martinčič 2009). Threat status: VU in Serbia, NT in Romania.

\section{Hypnum cupressiforme Hedw.}

COLLECTING SITES: 1 - bark of Abies; 2 - siliceous rock outcrops; 5 - limestone rock; 6 - limestone rock at stream; 9 - limestone and siliceous rock, and decaying wood; 10 - bark of Alnus; 12 - basic rock.

Hypnum cupressiforme Hedw. var. lacunosum Brid.

COLLECTING SITE: 2 - soil.
Isothecium alopecuroides (Lam. ex Dubois) Isov.

COLLECTING SITES: 1 - soil; 2,7 - siliceous rock; 5 limestone rock and bark of Fagus; 9 - limestone and siliceous rock, and bark of Fagus; 10 - bark of Alnus.

Isothecium alopecuroides (Lam. ex Dubois) Isov. var. robustum (Schimp.) Düll

COLlECTING SITE: 12 - wet meadow.

*Kieria starkei (F. Weber \& D. Mohr) I. Hagen

COLleCting SITE: 7 - siliceous rock outcrops (conf. E. Maier, M. Reimann).

NotES. Subarctic, subalpine element (Düll 1984). In SE Europe known only from Bulgaria, Bosnia-Herzegovina, Romania and Slovenia.

Leptodon smithii (Hedw.) F. Weber \& D. Mohr COLLeCting Site: 5 - bark of Fagus.

Lescuraea saxicola (Schimp.) Molendo

COLLECTING SITE: 2 - siliceous rock outcrops (conf. E. Maier).

NotES. From Montenegro there are records from the Prokletije Mts (Martinčič 2006). Threat status: VU in Bulgaria and Romania.

Leucodon sciuroides (Hedw.) Schwägr.

COLLECTING SITES: 2, 5, 9 - bark of Fagus; 10 - bark of Alnus.

Mnium lycopodioides Schwägr.

COLLECTING SITE: 1 - soil.

Notes. Apparently not rare in Montenegro (Papp and Erzberger 2010, 2011) and neighboring countries, e.g., Serbia (Papp \& Sabovljević 2002; Papp \& Erzberger 2005, 2007b). Threat status: VU in Bulgaria and Romania.

Mnium marginatum (Dicks.) P. Beauv.

COLLECTING SITES: 5, 8, 9 - limestone rock; 6 - limestone rock at stream; 9 - soil; 12 - soil near spring. 
Mnium spinosum (Voit) Schwägr.

COLLECTING SITE: 8 - limestone rock.

Mnium spinulosum Bruch \& Schimp.

COLLECTING SITE: 1 - soil.

Mnium stellare Hedw.

COLLECTING SITES: 8, 9 - limestone rock; 11 - schistose rock; 14 - soil among limestone rocks.

\section{Mnium thomsonii Schimp.}

COLLECTING SITES: 5, 8 - limestone rock; 6 - limestone rock at stream.

\section{Myurella julacea (Schwägr.) Schimp.}

COLLECTING SITE: 2 - limestone rock.

Notes. Apparently not rare in Montenegro (Papp \& Erzberger 2007a, 2010; Papp et al. 2008) nor in Serbia (Papp \& Erzberger 2007b, 2009; Papp et al. 2004). Threat status: LR in Montenegro and Serbia, VU in Romania.

Neckera besseri (Lobarz.) Jur.

COLleCtiNG SITE: 5 - limestone rock and bark of Fagus.

\section{Neckera complanata (Hedw.) Huebener}

COLLECTING SITES: 1 - bark of Abies; 5 - bark of Fagus; 9 - limestone rock and bark of Fagus; 10 - bark of Alnus.

Neckera crispa Hedw.

COLlECTING SITES: 5, 9 - limestone rock.

Neckera menziesii Drumm.

COLlecting SITES: 5 - limestone rock and bark of Fagus; 9 - bark of Fagus.

Neckera pumila Hedw.

COLLECTING SITE: 10 - bark of Alnus incana.

NoTES. From Montenegro there are a record from Morača gorge (Pavletić \& Pulević 1980) and recent collections from the Durmitor Mts (Papp
\& Erzberger 2010) and Orjen Mts (Papp et al. 2008). Threat status: DD in Montenegro, VU in Romania, EN in Bulgaria.

Orthothecium intricatum (Hartm.) Schimp.

COLLECTING SITE: 5 - limestone rock.

Orthotrichum cupulatum Hoffm. ex Brid.

COLLECTING SITE: 2 - siliceous rock.

*Orthotrichum cupulatum Hoffm. ex Brid. var. riparium Huebener

COLleCting SITES: 5, 6 - limestone rock in stream.

NotES. Sub-Atlantic taxon (Düll 1985). The SE European checklist of mosses mentions no varieties of Orthotrichum cupulatum (Sabovljević et al. 2008) but Düll et al. (1999) report this variety from Bosnia-Herzegovina, Croatia and Slovenia; also known from Greece (Papp et al. 1998), Serbia (Papp et al. 2006; Papp \& Erzberger 2009) and Albania (Papp et al. 2010).

Orthotrichum lyellii Hook. \& Taylor

COLLeCting SiTES: 2 - bark of Fagus; 10 - bark of Alnus.

Orthotrichum pallens Bruch ex Brid.

COllecting SITES: 1 - bark of Salix caprea; 2, 9 bark of Fagus.

Orthotrichum pumilum Sw. ex anon.

COLLECTING SITES: 2 - siliceous rock and bark of Fagus; 5 - bark of Sambucus nigra.

Orthotrichum rupestre Schleich. ex Schwägr.

COLLECTING SITES: 2 - siliceous rock and bark of Fagus; 5 - bark of Fagus; 6 - limestone rock at stream.

\section{Orthotrichum speciosum Nees}

COLLeCTING SITE: 1 - bark of Salix caprea and Abies.

Orthotrichum stramineum Hornsch. ex Brid.

COLleCting SITES: 1 - bark of Abies; 2, 5 - bark of Fagus; 9 - bark of Sorbus; 10 - bark of Alnus. 


\section{Orthotrichum striatum Hedw.}

COLlecting Sites: 1 - bark of Salix caprea and Abies; 2, 5 - bark of Fagus; 10 - bark of Alnus.

Oxystegus tenuirostris (Hook. \& Taylor)

A. J. E. Sm.

COllecting Sites: 1 - soil; 6 - limestone rock in stream; 10 - bark of Alnus.

\section{Palustriella commutata (Hedw.) Ochyra}

COLLECTING SITES: 4, 12, 16 - wet meadow; 5 - concrete and limestone rock in stream; 18 - peat in spring mire.

\section{Palustriella decipiens (De Not.) Ochyra}

COLlecting Sites: $11-$ schistose rock and soil at rivulet; 16 - wet meadow.

\section{Palustriella falcata (Brid.) Hedenäs}

COLLECTING SITES: 12,16 - wet meadow.

Paraleucobryum longifolium (Hedw.) Loeske

COLLECTING SITE: 7 - siliceous rock.

Paraleucobyrum sauteri (Bruch \& Schimp.)

Loeske

COLLECTING SITE: 2 - siliceous rock.

Notes. Threat status: R in the Red Data Book of European Bryophytes, VU in Montenegro and Bulgaria.

\section{Philonotis caespitosa Jur.}

COLLECTING SITE: 12 - siliceous rock and wet meadow; 18 - peat in spring mire.

Notes. From Montenegro there are two old records and a recent one (Dragićević \& Veljić 2006). Threat status: DD in Montenegro and Serbia, VU in Bulgaria.

Philonotis calcarea (Bruch \& Schimp.) Schimp.

COLlecting SITES: 4, 12 - peat in wet meadow and near spring.
Philonotis fontana (Hedw.) Brid.

COLLECTING SITES: 1 - at stream; 2 - at spring; 4 - wet meadow; 11 - schistose rock at rivulet; 12,16 - wet meadow; 18 - peat in spring mire.

Philonotis marchica (Hedw.) Brid.

COLLECTING SITE: 4 - wet meadow.

Notes. From Montenegro there are two old and two recent records according to Dragićević and Veljić (2006), and it was found in two localities in the Durmitor Mts (Papp \& Erzberger 2010). Threat status: EN in Bulgaria, NT in Romania.

Philonotis seriata Mitt.

COLleCting SITES: 11 - schistose rock at rivulet; 12 , 16,18 - wet meadow.

\section{Philonotis tomentella Molendo}

COLLECTING SITE: 4 - wet meadow.

Plagiobryum zierii (Hedw.) Lindb.

COLLECTING SITE: 2 - limestone rock.

Notes. From Montenegro there are two old (Dragićević \& Veljić 2006) and several recent records from the Orjen and Durmitor Mts. (Papp \& Erzberger 2010; Papp et al. 2008). Threat status: LR in Montenegro and Serbia, VU in Bulgaria.

Plagiomnium affine (Blandow ex Funck)

T. J. Kop.

COLLECTING SITES: 1 - soil; 14 - soil among limestone rocks.

Plagiomnium cuspidatum (Hedw.) T. J. Kop.

COLLECTING SITES: 9 - limestone rock; 10 - soil at stream.

Plagiomnium elatum (Bruch \& Schimp.)

T. J. Kop.

COLLECTING SITES: 1, 5 - soil at stream; 4 - wet meadow; 9 - limestone rock; 11 - schistose rock at rivulet. 
Plagiomnium rostratum (Schrad.) T. J. Kop.

COLLECTING SITES: 1 - soil; 2 - limestone rock and soil at spring; 5, 9 - limestone rock; 12, 16 - wet meadow.

\section{Plagiomnium undulatum (Hedw.) T. J. Kop.}

COLLECTING SITES: 1,9 - soil; 5 - limestone rock in stream; 10 - soil at stream.

Plagiopus oederianus (Sw.) H. A. Crum

\& L. E. Anderson

COLlecting SITES: 2, 5 - limestone rock.

Plagiothecium cavifolium (Brid.) Z. Iwats.

COLLECTING SITES: 8 - limestone and siliceous rock; 1, 9 - soil and limestone rock; 14 - soil among limestone rocks.

Plagiothecium denticulatum (Hedw.) Schimp. var. denticulatum

COLLecting SITE: 1 - soil.

Plagiothecium denticulatum (Hedw.) Schimp. var. undulatum R. Ruthe ex Geh.

COLLECTING SITE: 1 - soil.

\section{Plagiothecium platyphyllum Mönk.}

COLLECTING SITE: 9 - limestone rock.

NoTES. In Montenegro known from the Prokletije (Martinčič 2006) and Durmitor Mts (Papp \& Erzberger 2010) from one locality each. Threat status: VU in Bulgaria, NT in Romania.

\section{"Plagiothecium succulentum (Wilson) Lindb.}

COLLECTING SITES: 1, 7, 9 - soil; 2 - siliceous rock.

SubAtlantic element (Düll 1985). Known from many SE European countries, e.g., Bulgaria, Bosnia-Herzegovina, Greece, Romania, Serbia, Slovenia and the European part of Turkey. Recently also reported from Albania (Papp et al. 2010).

Plasteurhynchium striatulum (Spruce) M. Fleisch.

COLLECTING SITES: 5, 9 - limestone rock.
Platydictya jungermannioides (Brid.) H. A. Crum

COLLECTING SITE: 2 - limestone rock.

Notes. In Montenegro known from the Prokletije (Martinčič 2006) and Durmitor Mts (Papp \& Erzberger 2010) from one locality each. Threat status: VU in Bulgaria, NT in Romania.

Platyhypnidium riparioides (Hedw.) Dixon

COLLECTING SITES: 1 - at stream; 5, 6- limestone rock in stream; 12 - peat near spring.

Pleurozium schreberi (Willd. ex Brid.) Mitt.

COLLECTING SITE: 12 - soil.

Pogonatum aloides (Hedw.) P. Beauv.

COLlecting Sites: $1,7,9$ - soil.

Pogonatum urnigerum (Hedw.) P. Beauv.

Collecting Sites: 1, 7, 8, 9 - soil and soil among siliceous rock outcrops.

Pohlia andalusica (Höhn.) Broth.

COLleCting Sites: 13, 14 - soil among limestone rocks.

Notes. Reported recently for the first time in Montenegro (Erzberger \& Papp 2007); known from several localities in the Durmitor Mts (Papp \& Erzberger 2010).

Pohlia cruda (Hedw.) Lindb.

COLleCting SITES: $1,7,16$ - soil; 2 - siliceous and limestone rock; 8 - limestone rock and soil among siliceous rocks; 11 - schistose rock; 14 - soil among limestone rocks.

Pohlia melanodon (Brid.) A. J. Shaw

COLLECTING SITES: 1,16 - soil.

Pohlia wahlenbergii (F. Weber \& D. Mohr)

A. L. Andrews

COLlecting SITES: 1 - soil; 15 - at spring. 
Polytrichastrum alpinum (Hedw.) G. L. Sm.

COLleCting SITES: 1, 9, 16 - soil; 2, 8 - soil among siliceous and basic rocks; 11 - schistose rock; 14 limestone rock.

Polytrichum commune Hedw. var. commune

COLlecting SITE: 18 - peat in spring mire.

Polytrichum commune Hedw. var. perigoniale (Michx.) Hampe

COLlecting Site: 18 - peat in spring mire.

NoTES. Reported recently for the first time in Montenegro from the Durmitor Mts (Erzberger \& Papp 2007); this is the second record from the country.

Polytrichastrum formosum (Hedw.) G. L. Sm.

COLLECTING SITES: 8 - limestone rock; 1, 9 - soil; 11 schistose rock; 12 - peat near spring.

\section{Polytrichum juniperinum Hedw.}

COLLECTING SITES: 1, 7 - soil; 2 - soil among siliceous and basic rocks; 3,14 - soil among limestone rocks; 11 - soil among schistose rocks; 15 - acidic soil.

\section{Polytrichum piliferum Hedw.}

COLleCting SITES: 2, 7 - soil among siliceous rocks.

\section{Pseudoleskea incurvata (Hedw.) Loeske}

COLleCting SITES: 1, 2, 7, 8, 9 - soil among siliceous rocks; 11 - schistose rock; 13 - soil; 14 - limestone rock.

Pseudoleskea patens (Lindb.) Kindb.

COLleCting Sites: 2 - siliceous rock; 12 - basic rock.

Pseudoleskea radicosa (Mitt.) Macoun \& Kindb.

COLLECTING SITE: 2 - siliceous boulder.

Notes. From Montenegro there are several records from the Prokletije Mts (Martinčič 2006) and another old record from Andrijevica (Düll et al. 1999). Threat status: NT in Bulgaria and Romania.

\section{Pseudoleskea saviana (De Not.) Latzel}

COLleCting SITES: 1, 7, 9 - siliceous rock, 11 - schistose rock.

NOTES. Not rare in Montenegro (Martinčič 2006; Papp \& Erzberger 2010, 2011) and in Serbia (Papp \& Erzberger 2005, 2007b; Papp et al. 2004, 2012). Threat status: RT in the Red Data Book of European Bryophytes, VU in Montenegro and Serbia, CR in Romania.

Pseudoleskeella catenulata (Brid. ex Schrad.) Kindb.

COLLECTING SITES: 1 - siliceous rock; 9 - limestone rock.

Pseudoleskeella nervosa (Brid.) Nyholm

COLLECTING SITE: 2 - bark of Fagus.

Pseudotaxiphyllum elegans (Brid.) Z. Iwats.

COLLECTING SITES: 7 - siliceous rock; 9 - soil.

NoTES. Reported as new for the Montenegrin bryophyte flora based on this collection (Erzberger et al. 2008). Threat status: VU in Bulgaria, NT in Romania. Probably under-collected. From neighboring Serbia there are several recent records (Papp \& Erzberger 2005, 2007b, Papp et al. 2012).

\section{Pterigynandrum filiforme Hedw.}

COLLECTING SITES: 1 - siliceous rock and bark of Abies; 2, 9- siliceous rock and bark of Fagus; 5 - bark of Sambucus nigra; 7 - siliceous rock; 10 - bark of Alnus.

Ptychodium plicatum (Schleich. ex F. Weber \& D. Mohr) Schimp.

COLleCting SITES: 8,14 - limestone rock; 11 - schistose rock.

Notes. From Montenegro there are an old record from Komovi Mts (Velenovsky 1901; Dragićević \& Veljić 2006) and several recent records from the Prokletije Mts (Martinčič 2006); also known from the Durmitor Mts (Papp 
\& Erzberger 2010). Threat status: VU in Bulgaria and Romania.

Racomitrium aciculare (Hedw.) Brid.

COLLECTING SITES: 1, 2 - siliceous rock.

*Racomitrium affine (F. Weber \& D. Mohr) Lindb.

COLLECTING SITE: 1 - siliceous rock (conf. H. BednarekOchyra).

NoteS. Boreal, montane species (Düll 1984). Known in SE Europe only from Bulgaria, Romania (Sabovljević et al. 2008) and Greece (Papp et al. 2011).

\section{${ }^{*}$ Racomitrium aquaticum (Brid. ex Schrad.)} Brid.

COLleCting SITE: 1, 17 - siliceous rock; 11 - basic rock.

NoTES. Boreal, montane element (Düll 1984). Known in SE Europe in many countries, e.g., Bulgaria, Bosnia-Herzegovina, Croatia, Greece, Romania and Slovenia.

Racomitrium canescens (Hedw.) Brid.

COLLECTING SITES: 2,7 - siliceous rock; 3 - soil among limestone rocks; 13 - soil near late snow bed (in part conf. H. Bednarek-Ochyra).

\section{Racomitrium elongatum Ehrh. ex Frisvoll}

COLLECTING SITES: 12 - soil among siliceous rocks; 2 , 15 - acidic soil; 18 - soil (in part det./rev. H. BednarekOchyra).

Notes. Apparently not rare in Montenegro, with several recent records from the Durmitor and Prokletije Mts (Kürschner \& Parolly 1997; Dragićević \& Veljić 2006; Martinčič 2006; Papp \& Erzberger 2010). Threat status: DD in Montenegro.

Racomitrium lanuginosum (Hedw.) Brid.

COLLECTING SITES: 7 - siliceous rock; 11 - schistose rock.
NOTES. Reported as new for the Montenegrin bryophyte flora based on part of this collection (site 7: Erzberger et al. 2008).

Racomitrium sudeticum (Funck) Bruch \& Schimp.

COLLECTING SITES: 7, 8, 17 - siliceous rock; 11 -schistose rock; 13 - siliceous rock near late snow bed.

Rhizomnium punctatum (Hedw.) T. J. Kop.

COLLECTING SITES: 1 - soil, stones and decaying wood; 9 - base of Fagus and decaying wood; 10 - soil at stream; 11 - schistose rock at rivulet; 12,16 - wet meadow.

Rhytidiadelphus loreus (Hedw.) Warnst.

Collecting SITES: $1,7,16$ - soil.

Rhytidiadelphus squarrosus (Hedw.) Warnst.

COlLeCting SITE: 16 - soil.

Rhytidiadelphus triquetrus (Hedw.) Warnst.

COLlecting SiTes: 1,18 - soil; 2 - decaying wood; 8 - soil among siliceous rock outcrops.

Rhytidium rugosum (Hedw.) Kindb.

COLLECTING SITE: 13 - soil among limestone rocks.

Saelania glaucescens (Hedw.) Broth.

COLLECTING SITES: 2 - limestone rock; 8 - siliceous rock; 14 - soil among limestone rocks.

Sanionia uncinata (Hedw.) Loeske

COLLECTING SITES: 1 - decaying wood; 8,14 - limestone rock; 7, 15 - acidic soil; 12 - peat near spring; 13 - soil near late snow bed.

Schistidium agassizii Sull. \& Lesq.

COLLECTING SITE: 13 - soil near late snow bed.

Notes. From Montenegro there is an old record from the Durmitor Mts (Martinčič 1964). Threat status: VU in Montenegro and EN in Romania. 
Schistidium apocarpum (Hedw.) Bruch \& Schimp.

COLLECTING SITES: 1 - siliceous boulder; 3 - limestone rock; 5 - siliceous boulder near stream; 6 - limestone rock in stream; 9 - siliceous and basic rock.

Schistidium brunnescens Hedw. subsp. brunnescens

COLLECTING SITES: 14,16 - limestone rock.

Notes. It is a frequent Schistidium taxon in limestone areas. In Montenegro known from the Orjen Mts (Papp et al. 2008). There are several records from neighboring countries, e.g., Serbia (Papp \& Erzberger 2005, 2009), Albania (Papp et al. 2010). Threat status: NT in Bulgaria, EN in Romania.

Schistidium brunnescens Hedw. subsp. griseum

Collecting Sites: 2, 3 - basic rock (both conf. W. Schröder).

Notes. Reported as a new species for the Montenegrin bryophyte flora from the Lovćen Mts by Erzberger and Papp (2007); also collected in the Orjen (Papp et al. 2008) and Durmitor Mts (Papp \& Erzberger 2010). Apparently widespread on limestone rocks in the Balkans.

Schistidium confertum (Funck) Bruch \& Schimp.

COLlecting SITES: 2, 7, 8 - siliceous rock.

Notes. This is one of the most frequent Schistidium species on acidic rocks as evidenced by several recent collections from neighboring countries, e.g., Serbia (Papp \& Erzberger 2007b; Papp et al. 2012), FYR Macedonia (Papp \& Erzberger 2012; Papp et. al 2011) and Albania (Papp et al. 2010). From Montenegro there is one old record (Szepesfalvy 1931 - which cannot be accepted without revision) and a recent record (Dragićević \& Veljić 2006; Cvetić \& Sabovljević 2004). Threat status: NT in Bulgaria and Romania.

\section{Schistidium crassipilum H. H. Blom}

COLLECTING SITES: 1 - concrete; 5, 9 - limestone and other basic rock; 6 - limestone rock in stream.
Schistidium dupretii (Thér.) W. A. Weber

COLLECTING SITE: 1 - siliceous rock and concrete; 11 , 14 - on limestone and other basic rock.

NoTES. Reported as new for the Montenegrin bryophyte flora based on part of this collection (Erzberger et al. 2008).

Schistidium elegantulum H. H. Blom

COLLECTING SITES: 2, 9 - limestone rock.

Schistidium papillosum Culm.

COLLECTING SITE: 2 - siliceous rock.

NOTES. Reported as new for the Montenegrin bryophyte flora based on this collection (Erzberger et al. 2008).

"Schistidium pruinosum (Wilson ex Schimp.)

G. Roth

COLLECTING SITES: 2,8 - siliceous rock.

Notes. In SE Europe it is recorded in Bulgaria, Serbia (Sabovljević et al. 2008), Albania (Papp et al. 2010), FYR Macedonia (Papp et al. 2012), Greece (Blockeel 2010) and Romania (Stefanut \& Goia 2012).

Schistidium rivulare (Brid.) Podp.

COLleCting SITES: 1 - at stream; 11 - schistose rock at rivulet; 12 - siliceous rock at rivulet (in part conf. W. Schröder).

Notes. Threat status: CR in Montenegro.

Schistidium robustum (Nees \& Hornsch.)

H. H. Blom

COLLECTING SITES: 1, 5 - limestone rock.

NoTES. Reported recently for the first time in Montenegro (Erzberger \& Papp 2007); known from several localities in the Durmitor Mts (Papp \& Erzberger 2010).

Schistidium umbrosum (J. E. Zetterst.) H. H. Blom

COLLeCting SiTE: 8 - siliceous rock (det. H. Köckinger). 
Notes. Reported as new for the Montenegrin bryophyte flora based on this collection (Erzberger et al. 2008). Blom (1996) mentions a single specimen from SE Europe collected in Bosnia-Herzegovina.

"Sciuro-hypnum flotowianum (Sendtn.) Ignatov \& Huttunen

COLLECTING SITE: 9 - siliceous rock.

NoTES. This subcontinental montane element (Düll 1985) is known in SE Europe in many countries, e.g., Bulgaria, Bosnia-Herzegovina, Greece, Romania, Serbia and Slovenia (Sabovljević et al. 2008). Recently reported from Croatia (Papp et al. 2013).

Sciuro-hypnum populeum (Hedw.) Ignatov \& Huttunen

COLLECTING SITE: 1 - siliceous rock.

Scorpidium cossonii (Schimp.) Hedenäs

COLLECTING SITE: 12 - peat in wet meadow.

Scorpidium revolvens (Sw. ex anon.) Rubers

COLLECTING SITE: 12 - peat in wet meadow.

Sphagnum capillifolium (Ehrh.) Hedw.

COLlecting SITE: 18 - peat in spring mire.

Notes. All Sphagna are red-listed (VU) in Montenegro and Serbia, because of the limited extension and threatened status of suitable wetlands.

\section{Sphagnum contortum Schultz}

COLleCting SITE: 12 - peat near spring.

Notes. Threat status: VU in Montenegro and Serbia.

\section{Sphagnum russowii Warnst.}

COllecting SITE: 18 - peat in spring mire.

NotES. Threat status: VU in Montenegro and Serbia.

\section{Sphagnum squarrosum Crome}

COLleCting SITE: 18 - peat in spring mire.

Notes. Threat status: VU in Montenegro and Serbia.

Sphagnum teres (Schimp.) Ĺngstr.

COLLECTING SITE: 18 - peat in spring mire.

Notes. Threat status: VU in Montenegro and Serbia.

\section{Syntrichia norvegica F. Weber}

COLLECTING SITES: 2 - siliceous rock; 11 - schistose rock at rivulet.

Notes. Several recent records from high mountain areas of Montenegro, e.g., Orjen (Papp et al. 2008) and Durmitor Mts (Papp \& Erzberger 2010), and from FYR Macedonia (Papp \& Erzberger 2012; Papp et al. 2011). Threat status: LR in Montenegro and Serbia, NT in Bulgaria, VU in Romania.

Syntrichia ruralis (Hedw.) F. Weber \& D. Mohr

COLLECTING SITES: 1 - concrete and siliceous rock; 2 , 3 - siliceous rock and bark of Fagus; 3 - soil among limestone rocks; 5 - bark of Fagus; 6 - limestone rock in stream; 8 - limestone rock; 9 - limestone and siliceous rock, and bark of Fagus.

\section{Syntrichia virescens (De Not.) Ochyra}

COLLECTING SITES: 2 - siliceous rock and bark of Fagus; 5 - bark of Sambucus nigra.

Notes. Reported as new for the Montenegrin bryophyte flora based on part of this collection (site 2: Erzberger et al. 2008). Threat status: VU in Bulgaria, LR in Serbia. Probably overlooked and under-collected. From Montenegro these are the only known sites, but from neighboring countries there are several recent records, e.g., Serbia (Papp et al. 2004, 2006; Papp et al. 2007b), Albania (Papp et al. 2010) and FYR Macedonia (Papp \& Erzberger 2012). 
Tetraphis pellucida Hedw.

COLLECTING SITES: $1,9,10$ - decaying wood.

NotES. Red-listed (VU) in Montenegro and Serbia. In Montenegro one previous record from the Durmitor Mts (Martinčič 1964; Dragićević $\&$ Veljić 2006), but from Serbia there are several recent records (Papp and Erzberger 2005; Papp and Sabovljević 2002; Papp et al. 2004).

Thamnobryum alopecurum (Hedw.) Gangulee

COLLECTING SITE: 5 - limestone rock.

Thuidium assimile (Mitt.) A. Jaeger

COLLECTING SITES: 5, 9 - limestone rock.

Timmia austriaca Hedw.

COLLECTING SITES: 2, 8, 14 - limestone rock.

Tortella fragilis (Hook. \& Wilson) Limpr.

COLLECTING SITE: 2 - limestone rock.

Tortella tortuosa (Hedw.) Limpr.

COLLECTING SITES: 2 - siliceous and limestone rock; 3 , 14 - soil among limestone rocks; 5, 9, 16 - limestone rock; 10 - bark of Alnus; 11 - schistose rock.

Tortella tortuosa (Hedw.) Limpr. var. fragilifolia (Jur.) Limpr.

COLLECTING SITES: 2 - siliceous rock; 16 - limestone rock.

\section{Tortula hoppeana (Schultz) Ochyra}

Collecting Sites: 2, 3, 14 - soil among limestone and other basic rocks; 7 - siliceous rock; 8 - limestone rock.

Tortula muralis Hedw.

COLLECTING SITE: 1 - concrete.

Tortula schimperi M. J. Cano, O. Werner \& J. Guerra

COLLECTING SITE: 1 - soil.
Tortula subulata Hedw.

COLLECTING SITES: 2 - siliceous rock; 9 - soil; 11 schistose rock.

Trichodon cylindricus (Hedw.) Schimp.

COLLECTING SITE: 2 - soil; 13 - soil among limestone rocks.

NOTES. Probably overlooked and under-collected. Threat status: VU in Bulgaria and Romania.

Ulota bruchii Hornsch. ex Brid.

COLleCting SITES: 1 - bark of Picea abies; 9 - bark of Alnus incana and Sorbus; 10 - bark of Alnus incana.

NotES. Reported as new for the Montenegrin bryophyte flora based on part of this collection (site 1: Erzberger et al. 2008).

Ulota crispa (Hedw.) Brid.

COLLECTING SITES: 9, 10 - bark of Alnus incana.

Warnstorfia exannulata (Schimp.) Loeske

COLLECTING SITES: 11 - schistose rock at rivulet; 12 , 18 - peat near spring and in spring mire.

Weissia controversa Hedw.

COLlecting SITE: 16 - soil.

Weissia controversa Hedw. var. crispata (Nees \& Hornsch.) Nyholm

COLLECTING SITE: 2 - soil.

Zygodon rupestris Schimp. ex Lorentz

COLLeCting SITE: 10 - bark of Alnus.

\section{CONCLUSIONS}

The high geological diversity and wide range of elevations in the Bjelasica Mts support a particularly rich bryophyte flora. Acidic bedrock is rather rare in Montenegro, as most mountain ranges are built of limestone, and therefore the Bjelasica Mts are of special importance for the bryophyte diversity of 
the country. This is underlined by the rich liverwort flora of this mountain range. Our records comprise more than $50 \%$ of the Montenegrin hepatic flora. Mountain climate and acidic habitats appear to be the key factors for this high liverwort diversity. For the same reasons, other boreal and subalpine elements also appear in the Bjelasica Mts, many of them rarities. Therefore this region should be designated an Important Bryophyte Area of the Balkans.

ACKNOWLEDGEMENTS. We are grateful to Daniel Vincek for his warm hospitality and interest in our work, and to Halina Bednarek-Ochyra, Beata Cykowska, Heribert Köckinger, Eva Maier, Markus Reimann, Wiebke Schröder, Sorin Stefanuț and Jiří Váňa for checking specimens.

\section{REFERENCES}

ANONYMOUS 1995. Red Data Book of European Bryophytes. European Committee for Conservation of Bryophytes. ECCB, Trondheim.

BECK G. \& SzyszyŁowicz I. 1888. Plantae a D.ore. Szyszylovicz in itinerere per Cernagoram et in Albania adjacente anno 1886 lectae. Typis Universitatis Jagellonicae, Cracoviae.

BLOCKEEL T. 2010. The bryophytes of Greece: new records and observations, 2. Nova Hedwigia Beih. 138: 129-146.

BLOM H. H. 1996. A revision of the Schistidium apocarpum complex in Norway and Sweden. Bryophyt. Biblioth. 49: $1-333$.

Cvetić T. \& SABovluević M. 2004. New and interesting bryophyte records from Montenegro. Phytologia Balcan. 10(2-3): 67-69.

DragićEvić S. \& Veljić M. 2006. Pregled mahovina Crne Gore. Prirodnjački Muzej Crne Gore, Podgorica.

DragićEviĆ S., PAPP B. \& ERzBERGER P. 2011. Distribution of Buxbaumia viridis (Moug. ex Lam. \& DC.) Brid. ex Moug. \& Nestl. (Bryophyta) in Montenegro. Acta Bot. Croat. 71(2): 1-6.

DÜLL R. 1983. Distribution of the European and Macaronesian liverworts (Hepaticophytina). Bryol. Beitr. 2: 1-115.

DÜLL R. 1984. Distribution of the European and Macaronesian mosses (Bryophytina) I. Bryol. Beitr. 4: 1-109.

DÜLL R. 1985. Distribution of the European and Macaronesian mosses (Bryophytina) II. Bryol. Beitr. 5: 110-232.

DÜLL R. 1995. Bryophytes of Greece. Bryol. Beitr. 10: $1-229$.
DÜll R., Ganeva A., MartinČIČ A. \& PAVletić Z. 1999. Contributions to the bryoflora of former Yugoslavia and Bulgaria. IDH-Verlag, Bad Münstereifel.

ERZBERGER P. \& PAPP B. 2007. New and noteworthy bryophyte records from Montenegro and Serbia. Willdenowia 37: 339-351.

ERZBERGER P., PAPP B. \& DragiĆEviĆ S. 2008. Notes on some newly recorded bryophytes from Montenegro. J. Bryol. 30: $167-170$.

Grolle R. \& LONG D. G. 2000. An annotated check-list of the Hepaticae and Anthocerotae of Europe and Macaronesia. J. Bryol. 22: 103-140.

Hill M. O., Bell N., Bruggeman-NANNENGa M. A., BruguÉS M., CANo M. J., EnRoth J., FlatberG K. I., Frahm J.-P., Gallego M. T., Garilleti R., Guerra J., HedenÄs L., Holyoak D. T., Hyvönen J., IGNATOV M. S., LARA F., MAZIMPAKA V., MuŃOZ J. \& SÖDERTSRÖM L. 2006. An annotated checklist of the mosses of Europe and Macaronesia. Bryological Monograph. J. Bryol. 28(3): 198-267.

Kaso G. \& HADŽIABLAhOvić S. 2008. National Park Biogradska gora. Natural characteristics. Tourist information. Centre for Ecology and Environmental Management, Republički zavod za zaštitu prirode Crne Gore. Podgorica.

KÜRsChNer H. \& PAROlly G. 1997. Additions to the bryophyte flora of the Durmitor National Park (Crna Gora) and a first conspectus of all records. Willdenowia 27: 249-264.

LAKUŠIĆ R. 1966. Vegetation of meadows and pastures on mountain Bjelasica. God. Biol. Inst. u Sarajevu 19: 25-186.

LOITLESBERGER K. 1905. Zur Moosflora der österreichischen Küstenländer. Verh. Zool.-Bot. Ges. Wien 55: 475-489.

MAIER E. 2010. The genus Grimmia Hedw. (Grimmiaceae, Bryophyta) a morphological-anatomical study. Boissiera 63: $3-377$.

MARKA J. \& SABOVLJEVIĆ M. 2011. New bryophyte records from Albania. J. Bryol. 33: 74-76.

MARKA J. \& XhUlaj M. 2011. Mosses from Lura region (Albania). International Journal of Ecosystems and Ecology Science 1(1): 27-30.

MARTINČIČ A. 1964. Beiträge zur Kenntnis der Moosflora Jugoslawiens. I. Durmitor (Montenegro). Biološki Vestnik 12: $43-49$.

MARTINČIČ A. 2003. Annotated check-list of the mosses of Slovenia. Hacquetia 2(1): 91-166 (in Slovenian with English summary).

MARTINČIČ A. 2006. Moss flora of the Prokletije mountains (Serbia and Montenegro). Hacquetia 5(1): 113-130.

MARTINČIČ A. 2009. Contributions to the bryophyte flora of Republic of Macedonia. Hacquetia 8(2): 97-114. 
Milikić S., Veljé M., Marin D. P. \& Petković B. 2001. Mosses of some springs of the Morača river basin. Archives of Biological Sciences, Belgrade 53(1-2): 45-49.

Natcheva R., Ganeva A. \& Spiridonos G. 2006. Red list of the bryophytes in Bulgaria. Phytologia Balcan. 12(1): 55-62.

Nebel M. \& Philippi G. 2000. Die Moose Baden-Württembergs, 1. Ulmer, Stuttgart.

PAPP B. \& ERZBERGER P. 2005. The bryophyte flora of GolijaStudenica Biosphere Reserve and some adjacent sites (SW Serbia, Serbia-Montenegro). Studia Bot. Hung. 36: 101-116.

PAPP B. \& ERZBERgER P. 2007a. Contribution to the bryophyte flora of Montenegro. Studia Bot. Hung. 38: 79-94.

PAPP B. \& ERZBERGER P. 2007b. Contribution to the bryophyte flora of western Stara Planina Mts (E. Serbia). Studia Bot. Hung. 38: 95-123.

PAPP B. \& ERZBERGER P. 2009. Contribution to the bryophyte flora of south-eastern Serbia: Suva Planina Mts and its surroundings. Studia Bot. Hung. 40: 125-142.

PAPP B. \& ERZBERger P. 2010. Contribution to the bryophyte flora of Durmitor National Park, Montenegro. Nova Hedwigia 138: 145-161.

PAPP B. \& ERZBERGER P. 2011. Additions to the bryophyte flora of the Tara river canyon and Durmitor area, Montenegro. Studia Bot. Hung. 42: 31-39.

PAPP B. \& ERZBERGER P. 2012. Contribution to the bryophyte flora of the Former Yugoslav Republic of Macedonia (FYROM). Polish Bot. J. 57(1): 205-221.

PAPP B. \& SABOVLJEVIĆ M. 2001. Contribution to the knowledge of the bryoflora of the region of Petnica (W Serbia, Yugoslavia). Studia Bot. Hung. 32: 107-120.

PAPp B. \& SABOVlJević M. 2002. The bryophyte flora of Tara National Park (W Serbia, Yugoslavia). Studia Bot. Hung. 33: 25-39.

Papp B., Erzberger P. \& SABovljević M. 2004. Contributions to the bryophyte flora of Kopaonik Mts (Serbia, Serbia-Montenegro). Studia Bot. Hung. 35: 67-80.

PAPp B., ERZBerger P. \& SABOVlJević M. 2006. Contribution to the bryophyte flora of Djerdap National Park (E. Serbia). Studia Bot. Hung. 37: 131-144.

PAPP B., ERZBERGER P. \& DRAGićEVIĆ S. 2008. Contribution to the bryophyte flora of the Orjen Mts, Montenegro. Studia Bot. Hung. 39: 101-112.

PApp B., ERzberger P. \& Marka J. 2010. Contribution to the bryophyte flora of eastern Albania (Korça and Kolonja Districts). Studia Bot. Hung. 41: 61-88.

Papp B., ERZBerger P. \& TSAKIRI E. 2011. Contribution to the bryophyte flora of Voras (Nidže) Mts (Greece and the
Former Yugoslav Republic of Macedonia). Studia Bot. Hung. 42: 51-76.

Papp B., SzURdoKi E. \& SABovljević M. 2012. Bryophyte flora of the Vlasina lake and its surrounding (SE Serbia). Studia Bot. Hung. 43: 27-45.

PAPP B., TSAKIRI E. \& BABALONAS D. 1998. Bryophytes and their environmental conditions at Enipeas (Mt. Olympos) and Lycorrema (Mt. Ossa) streams (Greece). In: I. TsEKoS \& M. Moustakas, Progress in Botanical Research: Proceedings of the 1st Balkan Botanical Congress, Dordrecht, Boston, pp. 129-132.

PAPP B., Alegro A., ŠEGOTA V., ŠAPIĆ I. \& VuKELIĆ J. 2013. Additions to the bryophyte flora of Croatia. J. Bryol. 35(2): 152-155.

PAVletić Z. \& PulEviĆ V. 1980. Prilog za briofitsku floru Crne Gore. CANU, Glasnik Odjjeljenja Prirodnih Nauka, Titograd 3: 111-131.

Radović M. \& Medenica S. 2006. Kolašin. Sustainable development. Kolašin Developmental Board, Kolašin.

Ros R. M., Mazimpaka V., Abou-Salama U., Aleffi M., Blockeel T. L., Brugués M., Cano M. J., Cros R. M., Dia M. G., Dirkse G. M., El SaAdawi W., ErdaĐ A., Ganeva A., GonZÁlez-MAncebo J. M., Herrnstadt I., KHALIL K., KÜRSCHNER H., LANFRANCO E., LOSADALima A., REFAI M. S., ROdRÍGEZ-NuńEZ S., SAbovljeVić M., SÉrgio C., Shabbara H., Sim-Sim M. \& SÖDERSTRÖM L. 2007. Hepatics and Anthocerotes of the Mediterranean, an annotated checklist. Cryptog. Bryol. 28(4): $351-437$.

Ros R. M., Mazimpaka V., Abou-Salama U., Aleffi M., Blockeel T. L., Brugués M., Cros R. M., Dia M. G., Dirkse G. M., Draper I., El SAADAWi W., ERdaĐ A., Ganeva A., Gabriel R., GonzÁlez-Mancebo J. M., Granger C., Herrnstadt I., Hugonnot V., Khalil K., KÜRschner H., Losada-Lima A., Luís L., Mifsud S., Privitera M., Puglisi M., SABOVlJević M., SÉRgio C., Shabbara H. M., Sim-Sim M., SotiauX A., TACChi R., VANDERPoORTEN A. \& WERnER O. 2013. Mosses of the Mediterranean, an annotated checklist. Cryptog. Bryol. 34(2): 99-283.

SABovljević M. \& NATChEVA R. 2006. A check-list of the liverworts and hornworts of Southeast Europe. Phytologia Balcan. 12: 169-180.

Sabovluević M., Cvetić T. \& Stevanović V. 2004. Bryophyte red list of Serbia and Montenegro. Biodiversity and Conservation 13: 1781-1790.

SABOVlJEVIĆ M., NATChEVA R., TSAKIRI E., DihorU G., DRAGIĆEVIĆ S., ERDAĞ A. \& PAPP B. 2008. Check-list of the mosses of SE Europe. Phytologia Balcan. 14: 207-244.

SCHIFFNER V. 1916 a. Hepaticae Latzelianae II. Ein Beitrag zur Kenntnis der Lebermoose Dalmatiens. Verh. Zool.-Bot. Ges. Wien 66: 186-201. 
SCHIFFNER V. 1916b. Hepaticae Baumgartnerianae dalmaticae. Oesterr. Bot. Z. 66: 1-21.

ŞTEFANUȚ S. \& GOIA I. 2012. Checklist and Red list of the Bryophytes of Romania. Nova Hedwigia 95(1-2): 59-104.
SzEPESFALVY J. 1931. Zur Moosflora Süd-Dalmatiens. Magyar Bot. Lapok 30: 137-146.

VelenovsKy J. 1901. Ein Beitrage zur Moosflora von Montenegro. Oesterr. Bot. Z. 70: 254-256.

Received 7 May 2013 